\title{
Healing trabecular microfractures in the bodies of lumbar vertebrae
}

\author{
B. VERNON-ROBERTS AND C. J. PIRIE \\ Bone and Joint Research Unit, Institute of Pathology, The London Hospital
}

The presence of nodular aggregates of woven bone situated on porotic trabeculae has been described in the patella in chondromalacia patellae (Darracott and Vernon-Roberts, 1971) and in the femoral head in osteoarthrosis and rheumatoid arthritis (Todd, Freeman, and Pirie, 1972). The distribution and microstructure of the nodular aggregates indicate that they may represent some form of new bone formation or buttressing effect at sites of trabecular stress (Darracott and Vernon-Roberts, 1971) or callus formation surrounding the sites of trabecular fractures (Todd and others, 1972). The presence of fractures through trabeculae with surrounding callus formed of woven bone has also been described in idiopathic aseptic necrosis of the femoral head (Lagier, 1971). The microstructure and distribution of these trabecular lesions in the patella and femoral head has stimulated us to search for similar lesions in the bodies of lumbar vertebrae, since diffuse or focal osteoporosis is a common finding in this region after the fifth decade, and since it is also clear that this region of the skeleton is constantly subjected to severe mechanical stresses throughout life. The possibility that such lesions could be concerned in the genesis of back pain was also considered when we started this study.

\section{Material and methods}

\section{SELECTION OF SPECIMENS}

Lumbar spines were obtained post mortem from 22 subjects selected from 100 consecutive post mortem examinations carried out at The London Hospital. The remaining 78 subjects were considered unsuitable for inclusion in this study because of malignant disease, chronic renal disease, gastrointestinal disease, Paget's disease, or obvious scoliosis. The 22 subjects comprised fifteen males of mean age 66 years (range 48 to 83 ) and seven females of mean age 69 years (range 37 to 89 ).

MACERATED SLICES OF LUMBAR SPINE

The lumbar vertebral bodies complete with intervening discs were removed by dividing the vertebral column by cutting through the intervertebral discs at T12/L1 and at
L5/S1 and by dividing the pedicles of the lumbar vertebrae. A slice $0.5-\mathrm{cm}$. thick was then cut from the central portion of the vertebral bodies and discs in the coronal plane using a band-saw. The marrow tissue was then washed out of the cancellous bone of the fresh slice using a fine jet of water. The specimen was then immersed in ethyl alcohol for $12 \mathrm{hrs}$ to remove fat, and any remaining soft tissues were carefully removed manually under the dissecting microscope. The bone was finally bleached by immersion in $10 \mathrm{vol}$. hydrogen peroxide solution for 12 hrs and then allowed to dry. Each macerated specimen (Fig. 1) was then placed on a black background under a thin glass plate upon which the outline of each vertebral body was traced. The specimen was then examined using क dissecting microscope, and the location and orientation of trabeculae bearing nodular lesions were marked on the overlying glass plate. After the specimen had been ex amined from both sides in this way, the location and orientation marks were transferred from the glass to a single outline tracing of an anterior view of the specimen on paper (Fig. 2).

RADIOLOGICAL EXAMINATION OF TRABECULAE Macerated slices of lumbar vertebrae, unmacerated fresh or fixed slices of lumbar vertebrae, and single or grouped trabeculae isolated by dissection from fresh, fixed, or macerated slices of lumbar vertebrae were radiographed in a Faxitron 8050-010 X-ray Inspection System (Field Emission U.K. Limited) using slow, very fine grain Ilford Line N4E.50 film which has very high contrast. The radiographs were examined microscopically and enlargements of selected fields were made by photographing the radiographs with a Carl Zeiss Tessovar Photomacrographic Zoom System which has fully automatic exposure control.

\section{MICROSCOPY OF TRABECULAR LESIONS}

From vertebral bodies in which large numbers of trabecular lesions were present, trabeculae bearing nodular aggregates were dissected from thin slices. In some cases the vertebral bodies had been fixed and decalcified beforehand; but, in others, trabeculae were removed from freshly washed vertebral slices and were then fixed in formolsaline. After suitable processing, the trabeculae were embedded in methyl methacrylate, $8 \mu \mathrm{m}$. sections were made, and the sections were stained with haematoxylin and eosin, thionin, and by the von Kossa technique. 


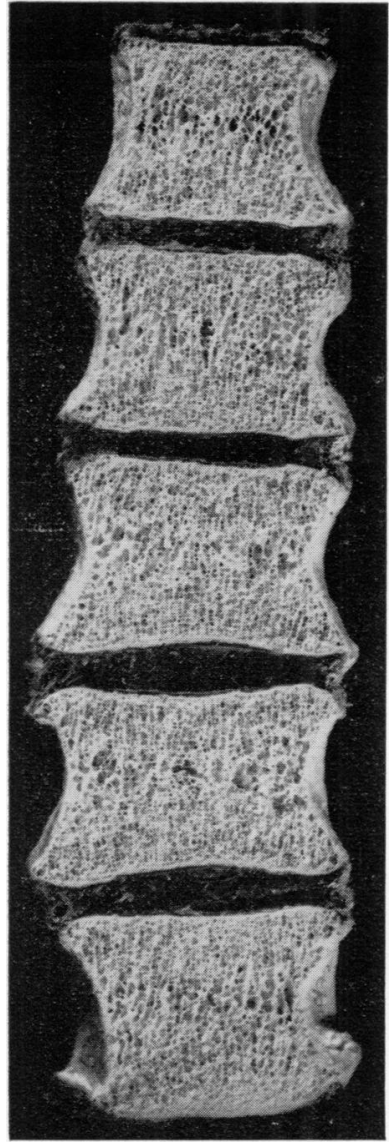

FIG. 1 Macerated coronal slab of lumbar bodies ready for examination under the dissecting microscope. The preparative procedure has distorted the normal disc structure

Results

FREQUENCY AND DISTRIBUTION OF

TRABECULAR LESIONS

Early in our examination of the macerated lumbar vertebral bodies, it became apparent that the trabecular lesions were predominantly situated in the upper and lower third of each vertebral body and were rarely found in the middle third. A composite diagram was therefore compiled (Fig. 3) in which each lesion seen in the 22 spines was denoted by a black spot placed in the appropriate position corresponding to the site of the lesion in the individual vertebral bodies. The affected trabeculae were indeed grouped in the upper and lower third of each vertebral body and relatively few lesions were present in the intervening middle third. However, although the lesions were usually situated in the region adjacent to the upper and lower subchondral osseus plates of each vertebral body, the vertical trabeculae directly con- nected to the osseus plates were rarely the site of trabecular lesions.

The aggregated total number of lesions present in each lumbar vertebral body from the 22 specimens increased from the lowest aggregated total in L1 to the highest in L4 (Fig. 3, 4; Table). The lower number of trabecular lesions in $\mathrm{L} 5$ relative to the numbers in L4 and L3 appears to be due to a reduction in the number of lesions in the lower part of the body of L5, and this could be the result of a variable degree of bone sclerosis which we have commonly found in the lower portion of L5.

The trabeculae of vertebral bodies are generally arranged in the vertical and horizontal planes in the erect subject. When trabeculae bearing nodular aggregates are divided into those on vertical trabeculae orientated at over $45^{\circ}$ to the erect horizontal plane, and those on horizontal trabeculae orientated at less than $45^{\circ}$ to the erect horizontal plane, it appears (Fig. 4; Table, overleaf) that the great majority of lesions are situated on vertical trabeculae. 
Table Distribution and number of trabecular lesions in bodies of lumbar vertebrae in 22 subjects

\begin{tabular}{|c|c|c|c|c|c|}
\hline \multirow[b]{2}{*}{ Vertebra } & \multicolumn{2}{|l|}{ Trabecula } & \multirow[b]{2}{*}{ Total } & \multirow[b]{2}{*}{ Range } & \multirow[b]{2}{*}{ Mean \pm S.E. } \\
\hline & $>45^{\circ}$ (vertical) & 45 (horizontal) & & & \\
\hline $\begin{array}{l}\text { L1 } \\
\text { L2 } \\
\text { L3 } \\
\text { L4 } \\
\text { L5 }\end{array}$ & $\begin{array}{r}77 \\
137 \\
132 \\
205 \\
136\end{array}$ & $\begin{array}{r}4 \\
12 \\
32 \\
14 \\
13\end{array}$ & $\begin{array}{r}81 \\
149 \\
164 \\
219 \\
149\end{array}$ & $\begin{array}{l}0-17 \\
0-26 \\
0-22 \\
0-36 \\
0-24\end{array}$ & $\begin{aligned} 4 & \pm 1 \\
7 & : 1 \\
7 & \pm 1 \\
10 & \pm 2 \\
7 & \pm 2\end{aligned}$ \\
\hline
\end{tabular}

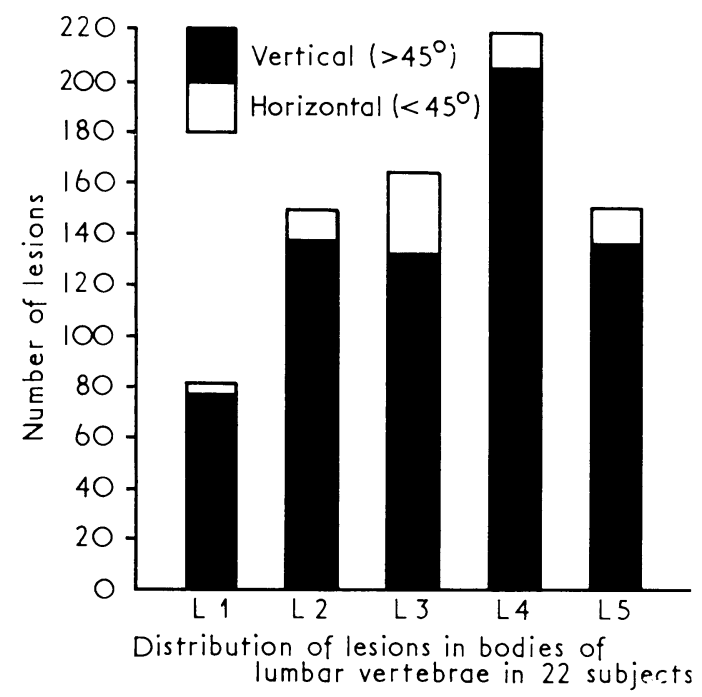

FIG. 4 Total number of lesions situated on vertical and horizontal trabeculae in all 22 spines examined. The number of lesions is greatest in $L 4$ and least in L1

The total number of lesions present in each of the 22 specimens varied from 2 to 112 . However, despite the wide range in the number of lesions in individual vertebral bodies examined overall (Table), there was no marked discrepancy in the numbers present in each of the five vertebral bodies in individual cases. The mean total number of lesions in each vertebral body was calculated (Table) and it was found that there was a significantly lower mean total in $\mathrm{Ll}$ in comparison with L4 $(\mathrm{P}<0.05)$ but not between all other means compared.

The degree of correlation between the total number of trabecular lesions in each specimen and such factors as age, height, weight, average disc thickness, and average depth of vertebral body has been calculated. The only statistically significant correlation obtained (Fig. 5) was between the total number of lesions and the ages of the 22 subjects $(r=0.65$; $\mathbf{P}<0.01)$. Fig. 5 shows that, in general, the number of lesions increases as age advances, particularly after the fifth decade. However, some of the subjects (all of

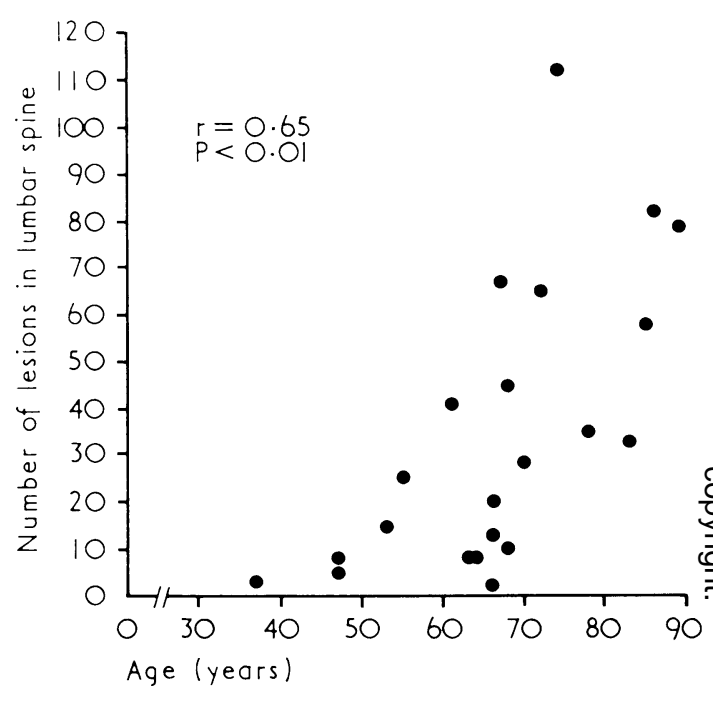

FIG. 5 Progressice increase in number of trabecular lesions in lumbar vertebral bodies as age advances. Each point represents the total number of lesions in one spine

whom were males) in the sixth decade had a total of less than twenty lesions, and it was observed that there was little evidence of osteoporosis in these specimens. Moreover, the higher numbers of lesions were seen in spines exhibiting marked diffuse osteoporosis. When taken in conjunction with our earlier observation that the number of lesions was reduced in the lower part of L5 when sclerosis of this region was present, it appeared that the frequency of the trabecular lesions was related to the degree of osteoporosis present. We also frequently observed that trabecular lesions were more numerous around or within areas of cancellous bone exhibiting a greater degree of osteoporosis than surrounding areas.

In some spines in which the lesions were numerous, they were occasionally disposed along vertical pathways in a chain-like manner (Fig. 6), and also tended to be grouped around the margins of Schmorl's nodes (Fig. 7) formed as the result of herniation of disc tissue through the bony end plate of the vertebral body. 
84.72
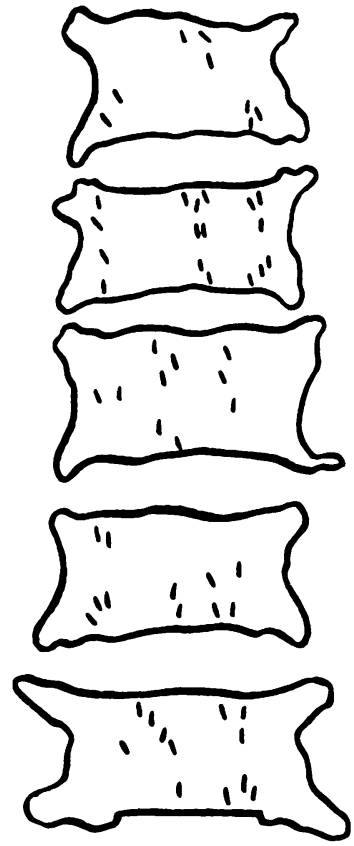

F 85

FIG. 6 Outline drawing of lumbar bodies, showing chainlike deposition of lesions on vertical trabeculae in a female aged 85 (Case 84/72)

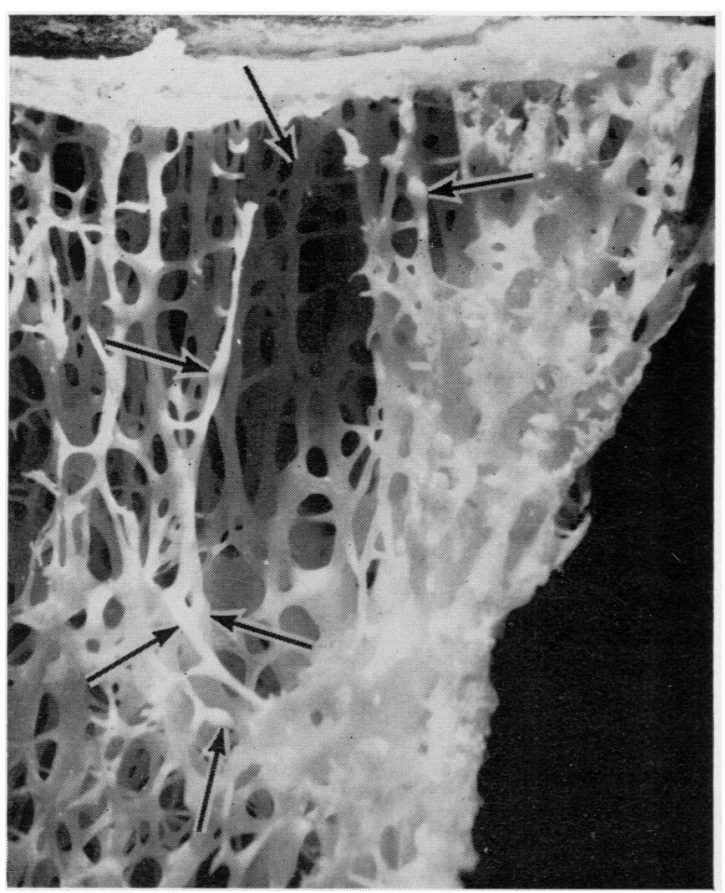

FIG. 7 Part of macerated fourth lumbar vertebra, showing defect in cancellous bone due to Schmorl's node, and number of trabecular lesions (arrows) in adjacent cancellous bone. $\times 5$
9
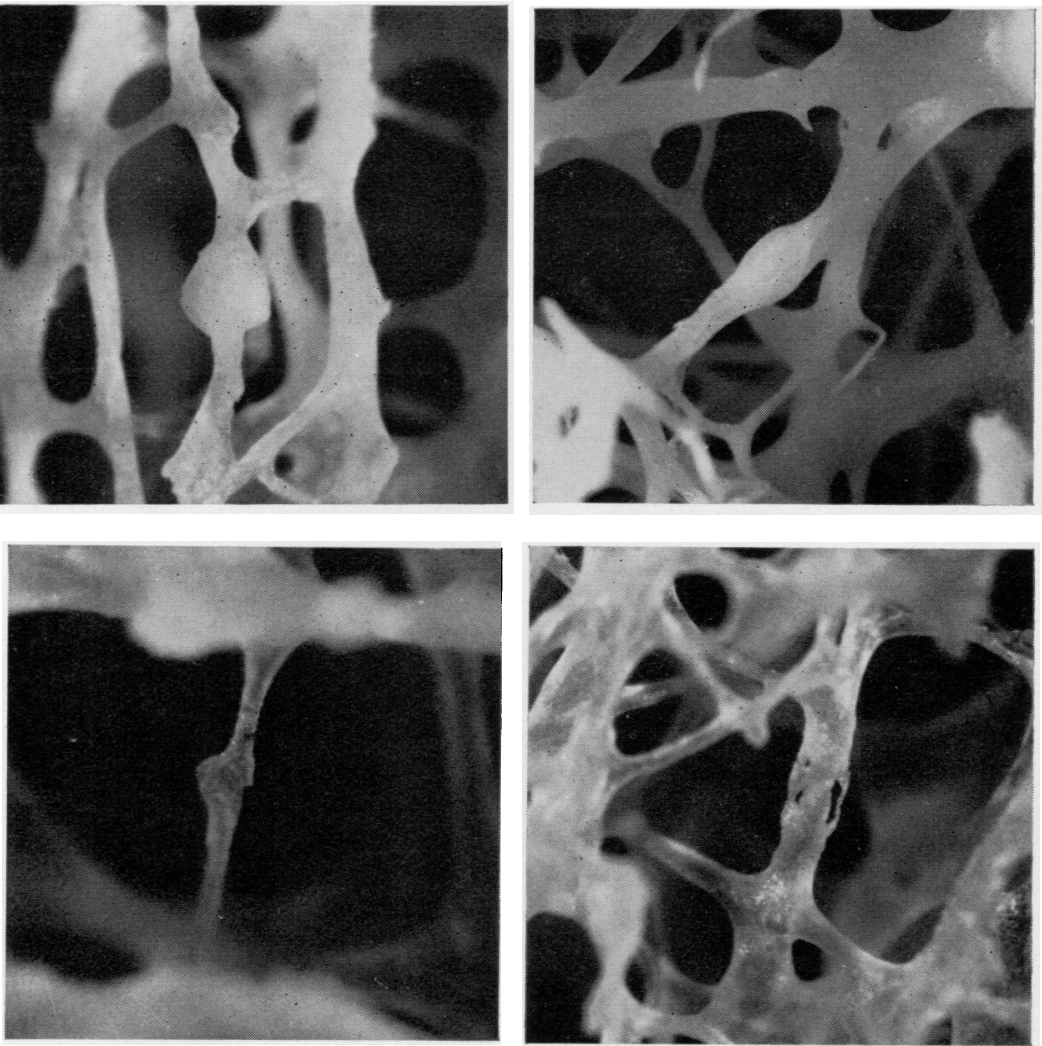

11

FIG. 8 Rounded nodular lesion on trabecula in macerated spine. $\times 20$

FIG. 9 Fusiform lesion on trabecula in macerated spine. $\times 20$

FIG. 10 Angulated excrescence on trabecula in macerated spine. $\times 20$

FIG. 11 Arched bridges on trabecula in macerated spine. $\times 20$

MICROSTRUCTURE AND $x$-RAY APPEARANCES OF TRABECULAR LESIONS

Under the dissecting microscope, the trabecular lesions vary in appearance from well-rounded discrete nodules up to $0.5 \mathrm{~mm}$. in diameter surrounding the affected trabecula (Fig. 8) to fusiform swellings (Fig. 9). Rarely the appearances are of angulated excrescences (Fig. 10) or delicate arched bridges (Fig. 11).

Microscopic examination of sections through trabecular lesions reveals that, in nearly all cases, they are composed of woven bone which has been laid down around the region of a transverse or oblique, partial or complete fracture through the underlying trabecula (Figs 12, 13, overleaf).

Enlargements made from high-resolution $x$ rays of intact trabecular lesions support the microscopic 


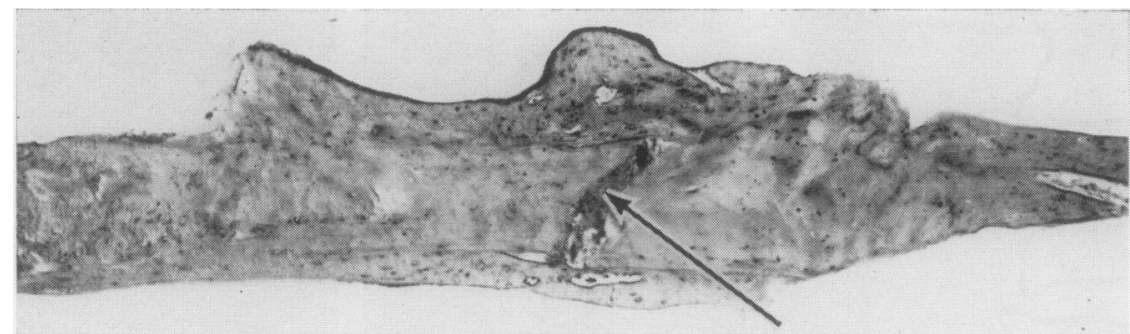

FIG. 12 Section through fusiform lesion on trabecula. Shows fracture through shaft of trabecula with debris (arrow) separating the broken ends of the trabecula, and new bone surrounding and uniting the fractured trabecula. Haematoxylin and eosin. $\times 100$

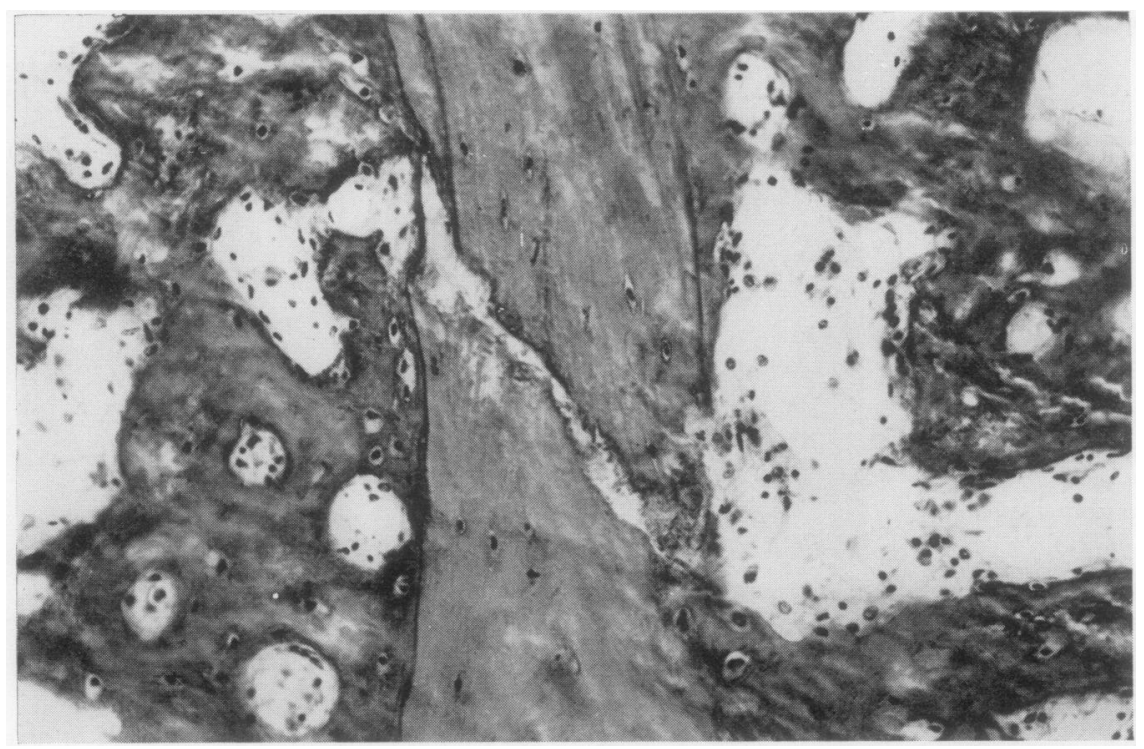

FIG. 13 Section through fractured trabecula, showing fracture site and surrounding woven bone. Haematoxylin and eosin. $\times 200$

findings that, in nearly all cases, the nodular aggregates of woven bone have formed at sites of trabecular fracture (Figs 14 and 15, opposite). In some cases, the appearances suggest a longitudinally-orientated crack rather than an oblique fracture line. In very few cases, radiological or microscopic evidence of abnormality in the underlying trabecular are absent.

\section{Discussion}

We have demonstrated the existence of nodular aggregates of woven bone situated on trabeculae in the bodies of lumbar vertebrae, and we have shown that these aggregates are in most cases formed in response to fracture of the affected trabeculae. They are structurally identical with similar nodular aggregates of woven bone situated on trabeculae previously described in the patella in chondromalacia patellae (Darracott and Vernon-Roberts, 1971), in the femoral head in osteoarthrosis and rheumatoid arthritis (Todd and others, 1972), and in idiopathic aseptic necrosis of the femoral head (Lagier, 1971).

We have shown that the trabecular lesions denoting microfractures are predominantly located in the upper and lower thirds of each lumbar vertebral body, and that the middle third is relatively free of such lesions. In this connection, it is of interest that, after repeated cyclic loading of intact vertebral bodies in a N mechanical simulator, Freeman and his colleagues found that fatigue fracture eventually occurred $N$ through the upper and lower thirds of the body and $\omega$ that the middle third is spared (M. A. R. Freeman, personal communication, 1972).

There is a statistically significant correlation between the number of trabecular lesions in the lumbar : spine and age, and we have also observed that the number of lesions is directly related to the degree of osteoporosis. It has been established that osteoporosis begins to develop in females over the age of 50 years, and affects both sexes equally over the age of 70 ? 
(a)

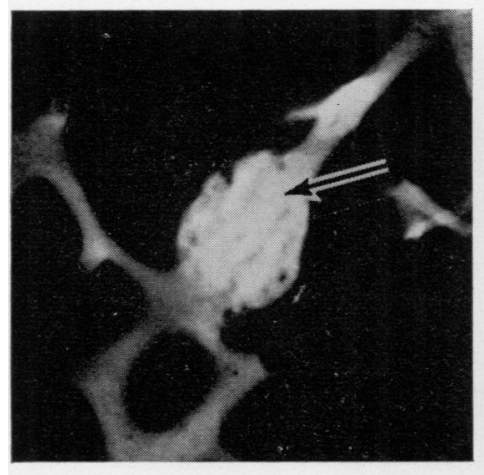

(b)



(c)

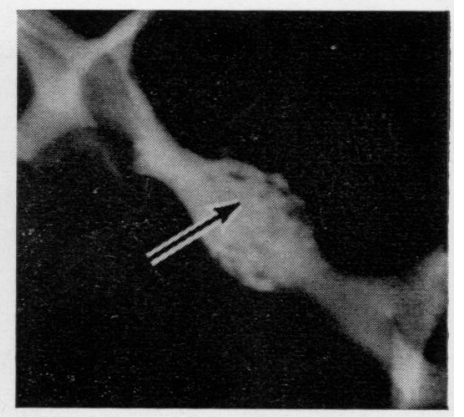

FIG. $14(a, b, c)$ Enlargements made from radiographs of isolated single trabeculae, showing defects suggesting fractures in trabeculae (arrows) and nodule of woven bone surrounding fracture site. $\times 30$

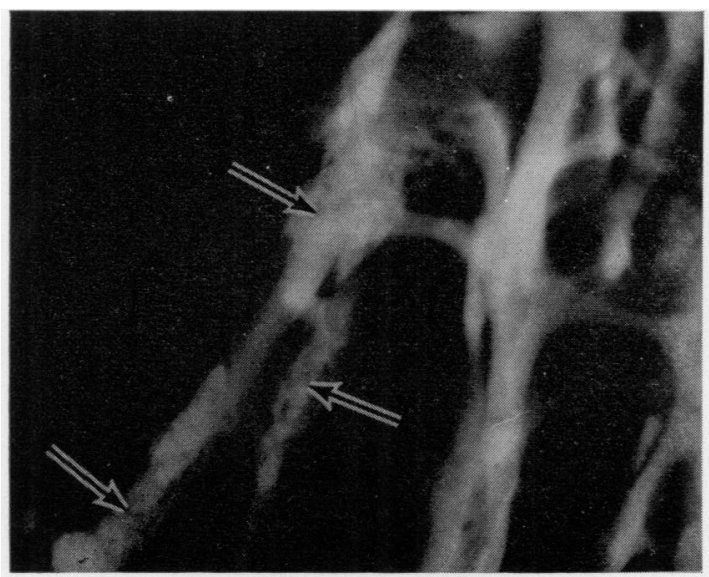

FIG. 15 Enlargement from radiograph of slice of vertebral body, showing defects in trabeculae suggesting fracture (arrows) and woven bone surrounding fracture sites. $\times 30$

(Nordin, 1971). It is thus of interest that, in the present study, we found that all five subjects between the ages of 60 and 70 who had less than a total of twenty trabecular lesions in the lumbar spine were male, and the one subject aged between 50 and 60 who had more than twenty lesions was female. We have not yet had the opportunity to examine the spine from a case of the crush-fracture syndrome, but it would be of interest to ascertain whether the reduced ability to form new bone which obtains in this syndrome (Nordin, 1971) is also evidenced by an absence the characteristic nodular aggregates formed in response to trabecular fracture.

We have found that the great majority of nodular aggregates formed in response to trabecular fracture are located on vertically-orientated trabeculae, and very few are present on horizontal trabeculae. In spinal osteoporosis there is preservation of vertical trabeculae and disappearance of the more horizontal ones (Atkinson, 1967; Bell, Dunbar, and Beck, 1967), and herein lies a possible explanation of the greater susceptibility of vertical trabeculae to fracture in osteoporotic vertebrae. According to the equation of von Euler, in a structure having cross-ties (horizontal trabeculae) dividing the length of the vertical trabeculae into two halves, the weight required to buckle the structure is inversely proportional to the square of the distance between the cross-ties (Bell and others, 1967); thus, in the absence of the cross-ties, the structure will buckle under one-quarter of the original load required. If the latter state of affairs applies to the situation which obtains to a greater or lesser degree in vertebral bodies in osteoporosis, one need not look further for an adequate explanation for the observed frequency of trabecular fractures occurring on vertical trabeculae in lumbar vertebral bodies in the ageing spine.

The observations presented in this paper concern only those trabeculae exhibiting callus formationusually in response to fracture. Although we have observed fractured trabeculae not showing evidence of callus formation, we have not attempted to quantitate these in the present study because of the difficulties of eliminating artefactual fractures produced during preparation of the specimens for examination.

The final question arises whether trabecular fractures can be a cause of back pain. While there is little doubt that sensory nerve fibres are present in cancellous bone, we are not at present in a position to confirm or deny that they may be involved either in the fracture of trabeculae or the repair process which gives rise to the nodular aggregates which we have described.

\section{Summary}

Lumbar spines were removed from 22 subjects at autopsy. The number and distribution of healing 
trabecular fractures was assessed in macerated slices of vertebral bodies, and the fractures were further examined by microscopy and radiography. Trabecular fractures were predominantly located on vertical trabeculae adjacent to the upper and lower subchondral osseus plates of vertebral bodies, and were increased in number in association with osteoporosis and advancing age.

We wish to record our thanks to Mr. M. A. R. Freeman and Dr. H. L. F. Currey for helpful discussion. We are grateful for financial support from the Arthritis and Rheumatism Council and the Medical Research Council

\section{References}

Atkinson, P. J. (1967) Calc. Tiss. Res., 1, 24 (Variation in trabecular structure of vertebrae with age)

Bell, G. H., DuNBAR, O., AND BECK, J. S. (1967) Ibid., 1, 75 (Variations in strength of vertebrae with age and their relation to osteoporosis)

DARracotT, J., AND VernON-RoBerTs, B. (1971) Rheum. phys. Med., 11, 175 (The bony changes in "chondromalacia patellae')

LAGIER, R. (1971) 'Idiopathic aseptic necrosis of the femoral head. An anatomo-pathological discussion of this concept', in 'Idiopathic Ischemic Necrosis of the Femoral Head in Adults', ed. W. M. Zinn, p. 49. Thieme, Stuttgart

NoRDIN, B. E. C. (1971) Brit. med. J., 1, 571 (Clinical significance and pathogenesis of osteoporosis)

Todd, R. G., Freeman, M. A. R., AND PIRIE, C. J. (1972) J. Bone Jt Surg., 54B, 723 (Isolated trabecular fatigue fractures in the femoral head) 